\title{
Desarrollo de competencias emprendedoras mediante iniciativas de aprendizaje basado en proyectos
}

\section{Development of Entrepreneurship Competences from Project Based Learning Approaches}

\author{
Lidia Sánchez González, Rubén Ferrero Castro, Miguel Ángel Conde González, Javier Alfonso \\ Cendón
}

Escuela de Ingenierías Industrial e Informática. Departamento de Ingenierías Mecánica, Informática y Aeroespacial. Campus de Vegazana S/N . 24071. León, España. \{lidia.sanchez, rferc, miguel.conde, javier.alfonso\}@unileon.es

\begin{abstract}
Resumen
Un aspecto clave para cualquier institución educativa es formar a los individuos para que se desenvuelvan eficientemente en la sociedad. En la actualidad el contexto en que se desarrolla la formación es la era digital. En este contexto y en la coyuntura socioeconómica actual, se hace fundamental incrementar la futura empleabilidad de los discentes. Esto requiere que los contenidos impartidos estén alineados con los requerimientos de las empresas, que los estudiantes sepan utilizar metodologías, técnicas y herramientas que les ayuden a estar mejor preparados para desempeñar sus futuros trabajos. En este sentido, uno de los aspectos más demandados por las empresas e instituciones es que los trabajadores no saben abordar un proyecto desde el principio. Dada esta situación, el presente artículo plantea la aplicación de una metodología de aprendizaje basado en proyectos para solventar este problema. En concreto, se desarrolla una experiencia durante dos años en los que los alumnos deben desarrollar proyectos completos en grupos y generar productos reales. Después de la experiencia se evalúa la adquisición de competencias y el incremento de la motivación del alumno y se observan resultados positivos en ambos sentidos.
\end{abstract}

\section{Palabras clave}

Aprendizaje basado en proyectos; aprender haciendo; emprendimiento; empleabilidad liderazgo.

\begin{abstract}
A key issue for any educational institution is to train individuals in such a way that they participate efficiently in their context. The current context where learning is carried out is digital era. With this landscape, and taking into account the current socioeconomic situation, it is necessary to increase students' employability. This requires that what is taught in educational institutions fits with companies' requirements. In this sense one of most common companies' requirement is that students have knowledge about how to develop a project from scratch. In order to address this issue, the present paper poses the application of a project based learning methodology. This methodology is applied in an experiment that comprises two academic years. During each year, the students should develop complete projects working as teams and obtain final products. In this way, it was possible to develop competences that can be interesting for companies and also to increment students' motivation.
\end{abstract}

\section{Keywords}

Project based learning; learning by doing; entrepreneurship; employability; leadership. 


\section{Introducción}

El Espacio Europeo de Educación Superior otorga gran importancia a la empleabilidad, indicando que los títulos deben preparar para el acceso al ejercicio profesional (González, 2008). Unas personas tienen más empleabilidad que otras, lo cual depende del propio individuo, de las empresas y de las tendencias del mercado (Michavila, Martín-González, Martínez, García-Peñalvo, \& Cruz-Benito, 2016).

En el Grado en Ingeniería Informática, las encuestas de nuevo ingreso muestran que la práctica totalidad de los estudiantes espera conseguir un trabajo como programador o informático, empleado por cuenta ajena en una empresa (Universidad-de-León, 2014). No hay un porcentaje, aunque sea mínimo, que planee crear su propia empresa. En este campo, que cambia a velocidades vertiginosas, es común ver en las noticias cómo pequeñas empresas (incluso un grupo de amigos) que desarrollan aplicaciones que triunfan entre los usuarios, se convierten en empresas muy rentables, o bien que son vendidas a los gigantes de las comunicaciones. A pesar de que la mayoría de los estudiantes conocen estos casos, no se ven a sí mismos capaces de conseguirlo.

Se hace por tanto necesario fomentar la transferencia de conocimiento entre la universidad y la empresa. Los estudiantes se enriquecerían de este modo, porque podrían medir su capacidad para satisfacer los requisitos de sus futuros empleadores y también al conocer que se supone que deben saber y qué se demanda desde el contexto empresarial. Además, de este modo las empresas podrían detectar el talento de los estudiantes y podrían tratar de amoldar sus procesos para formar profesionales adecuados (Clark, 1998; García-Peñalvo, 2016; Gomes-Pires, García-Peñalvo, MarinhoSampaio, \& Martínez-Vázquez, 2013; Vilalta, 2013).

En este sentido existen diferentes iniciativas que tratan de promover el intercambio de conocimiento con mayor o menor éxito (F. J. García-Peñalvo, Cruz-Benito, M. Á. Conde, \& Griffiths, 2014; GarcíaPeñalvo, 2015; García-Peñalvo, Cruz-Benito, Griffiths, \& Achilleos, 2015), y en esos casos siempre resulta beneficioso que los estudiantes hayan adquirido previamente competencias relacionadas con su futuro desempeño profesional.

Ante esta situación, el presente trabajo presenta una experiencia en la que seaplica una metodología con el fin de que los estudiantes adquieran las competencias necesarias para aumentar su empleabilidad, fomentando la pro-actividad, a través de la adquisición de competencias como: trabajo en equipo, desarrollo efectivo de la comunicación oral y escrita, la aplicación de sus conocimientos a su trabajo de forma profesional, etc. Para ello se ha aplicado el aprendizaje basado en proyectos.

El aprendizaje basado en proyectos (Project Based Learning o PBL) es un método docente que involucra al estudiante en el proceso de aprendizaje. El discente trata de solucionar un problema no trivial mediante 
el planteamiento de una serie de cuestiones que va refinando, el debate de posibles soluciones, la predicción de problemas, la definición de planes de trabajo, el análisis de datos, la elaboración de conclusiones y la comunicación de las ideas y los resultados obtenidos a otros. En los proyectos los elementos fundamentales son el problema a resolver y la generación de resultados conocidos como artefactos (Blumenfeld, et al., 1991).

La aplicación del PBL aporta ventajas como (Blank, 1997; Blumenfeld, et al., 1991; Bottoms \& Webb, 1998; Dickinson, et al., 1998; Musa, Mufti, Latiff, \& Amin, 2011; Rae, 2007; Thomas, 2000): incremento en la motivación de los estudiantes; incremento en la atención y la confianza en sí mismos; adquisición de competencias propias de la gestión del proyecto como elaboración de soluciones, gestión del tiempo, planificación; acerca al estudiante a problemas reales, lo que incrementa su empleabilidad; etc. Este método docente se aplica en el contexto de asignaturas de dirección de proyectos de ingeniería con éxito (Crespo, Alba-Elías, González-Marcos, Ordieres-Meré, \& Castejón-Limas; Jun, 2010) y en el contexto de esta investigación se utiliza para la asignatura de Arquitecturas Específicas y Empotradas, optativa de $4 .^{\circ}$ en la titulación de Grado en Ingeniería Informática desde que comenzó a impartirse en el curso 2014/15.

La adquisición de competencias se produce en su mayor parte con la práctica. El objetivo general del aprendizaje consiste en una meta a alcanzar, la cual se pueda evaluar con criterios de fiabilidad y validez. Esta evaluación de competencias tiene gran importancia, ya que influye en la formación inicial y continua, la cual es necesaria para el ejercicio de una profesión. Las competencias académicoprofesionales se construyen tanto en la Universidad como en el mundo laboral. En general, la mayoría del conocimiento factual (saber) se adquiere durante la formación universitaria y el conocimiento procedimental (saber hacer) se consigue en el desarrollo del trabajo (González, 2008).

La experiencia que se presenta en este trabajo trata de inculcar en los estudiantes una filosofía autodidacta y de continua formación, muy necesaria dentro de cualquier sector de la ingeniería. También pretende que adquieran competencias en la gestión de proyectos completos, resolución de problemas, prototipado rápido, etc., que fomentan actitudes proactivas y estimulan su motivación hacia los proyectos innovadores y el autoempleo.

A continuación, se va a describir la experiencia llevada a cabo en profundidad. Posteriormente, se presenta cómo se evalúa la experiencia y los resultados de la misma y por último se aportan una serie de conclusiones.

\section{Contexto Experimental}

En la asignatura optativa de Arquitecturas Específicas y Empotradas se aplica la metodología PBL 
de cara a la adquisición y evaluación de competencias de la asignatura, así como para aumentar la empleabilidad de los estudiantes. Dicha asignatura se ha impartido dos cursos académicos con unos 15 alumnos matriculados cada año. Para ello, la asignatura se ha organizado de la siguiente manera:

- Inicialmente, se realizan unas prácticas guiadas, supervisadas por los profesores de la asignatura, en las que se introducen a los alumnos los conceptos de sistemas empotrados.

- A continuación, se realiza el montaje de un dispositivo.

- Por último, se propone a los estudiantes que desarrollen en grupos de 2-4 personas un sistema empotrado plenamente funcional.

Para conseguir el objetivo perseguido por la experiencia se pretende obtener una serie de resultados de aprendizaje. Estos consisten en que el estudiante aprenda a: trabajar en equipo; analizar y resolver problemas; interpretar resultados y datos relevantes; comunicarse vía oral y escrita; aplicar los conocimientos de forma profesional; elaborar y defender argumentos; y saber emitir juicios sobre temas de diversa índole. Estos resultados implican la aplicación de una metodología, el uso de unos recursos y el planteamiento de unas actividades que se describen a continuación.

\subsection{Marco metodológico}

La metodología docente seguida implica crear el contexto necesario para poder conseguir los resultados de aprendizaje anteriores. Para ello, los profesores han de:

- Coordinar el uso de laboratorios buscando colaboración con otras áreas y profesores.

- Preparar materiales docentes que sirvan de guía a los estudiantes para desempeñar con éxito sus tareas.

- Supervisar el desarrollo de los proyectos en todo momento, para poder orientar a los estudiantes y favorecer la consecución de sus objetivos.

- Colaborar en la búsqueda de soluciones, en la toma de decisiones y ayudar en la adopción de las soluciones consensuadas por los grupos.

La metodología se basa en el modelo integrado de efectividad del trabajo en equipo, IMO (Viles Diez, Zárraga-Rodríguez, \& Jaca García, 2013), desarrollado para entornos organizacionales no académicos, pero aceptado en el ámbito académico como marco conceptual válido y de alto valor. Este modelo representa el trabajo en equipo como un conjunto de procesos condicionados por unos factores previos que dan lugar a unos resultados. Además, incorpora el carácter cíclico y dinámico, ya que los resultados se convierten en nuevas entradas del proceso. El modelo mencionado se ha adaptado y completado para incluir otras competencias y fomentar la empleabilidad de los estudiantes. En concreto, el trabajo propuesto por Viles et al. (2013) emplea un cuestionario para retroalimentar a los equipos sobre su desempeño. Para ello, creaban un gráfico radar que denominan "huella" con la escala de valoración para cada proceso operativo. De esta manera, cada equipo podía analizar las fortalezas 
y debilidades. Sin embargo, en esta experiencia, se ha empleado ese cuestionario para valorar el desempeño de los equipos de trabajo, de manera que se pueda calificar a los distintos estudiantes de acuerdo con el trabajo desarrollado. En cuanto a la realimentación, como la asignatura tiene pocos alumnos y el trato con el profesor es diario, se les comunicaba verbalmente aquellas carencias que se observaban en las sesiones de laboratorio y tutorías, considerando los aspectos detallados en el apartado "Observación directa". De esta forma, la retroalimentación era inmediata.

En cualquier caso, en esta metodología la adquisición de tales competencias viene condicionada por los factores previos y los mediadores (Viles Diez, et al., 2013).

\subsection{Herramientas y recursos}

En esta experiencia se ha hecho fundamental la disponibilidad de diferentes recursos. En concreto se va a diferenciar entre recursos humanos, estructurales, documentales y económicos.

En cuanto a los recursos humanos debe considerarse que, como la experiencia tiene un carácter multidisciplinar, han colaborado diversos profesores en las distintas etapas de la misma. En concreto, y más allá de los estudiantes involucrados en la experiencia, se ha contado con la ayuda de 11 docentes, un oficial de laboratorio y un alumno. 6 de los profesores han facilitado y adecuado instalaciones y equipos para que se pudiera llevar a cabo la experiencia. Otros 2 dan soporte como expertos en PBL y en evaluación de competencias. Los 3 restantes se encargan de impartir la asignatura y por tanto supervisan y conducen la experiencia, son estos los que llevan a cabo la mayor parte del trabajo. E oficial de laboratorio da soporte a la realización de los montajes y el alumno aporta su experiencia al haber realizado un trabajo de fin de grado relacionado con las actividades que se planteaban.

En lo que respecta a los recursos estructurales para el desarrollo de la asignatura y la experiencia descrita, se han utilizado espacios de la Escuela de Ingenierías Industrial e Informática. En concreto: un laboratorio para el montaje de impresoras 3D y escáner 3D (equipado con todo tipo de herramienta); un laboratorio para la impresión y escaneo de piezas en 3D, montaje de componentes de los proyectos, trabajo en grupo, etc. (dicho laboratorio se ha habilitado para el libre acceso a los alumnos); y otros dos laboratorios con equipos informáticos y tomas con IP pública.

Como recursos documentales, la asignatura proporciona en su guía docente una bibliografía recomendada. Sin embargo, la que los estudiantes han encontrado más útil ha sido la recogida en la plataforma moodle: prácticas guiadas para su iniciación en el uso de plataformas como Arduino o Raspberry Pi; y diversos manuales de montaje de impresoras 3D, escáner 3D, desarrollo y montaje de sistemas, etc. Se ha tratado de fomentar durante el transcurso de la experiencia la cultura del conocimiento abierto y los desarrollos colaborativos, por lo que otra gran fuente de documentación 
han sido los repositorios y blogs especializados en la red, donde se comparte todo tipo de información y documentación.

Otro aspecto nada desdeñable es el apartado de recursos económicos. En concreto, mediante el presupuesto de Actividad Académica del área de Arquitectura y Tecnología de Computadores, se han adquirido los componentes necesarios para el montaje y utilización de una impresora 3D y un escáner 3D. Además, se ha ido dotando de material necesario en el día a día para el desarrollo de los proyectos: kits de Arduino, Raspberry Pi, herramientas básicas, bobinas de filamento para la impresión, componentes como módulos de radiofrecuencia, bluetooth, shields, etc.

\subsection{Actividades}

Las actividades realizadas a lo largo de dos cursos académicos (2014/15 y 2015/16) en la asignatura de Arquitecturas Específicas y Empotradas se categorizan en:

- Actividades de adquisición de los conocimientos básicos. Los estudiantes realizan, bajo la supervisión de los profesores de la asignatura, un conjunto de prácticas guiadas para desarrollar pequeños sistemas basados en Arduino y Raspberry Pi. En ellas, se realizan montajes y se programan para conseguir pequeños dispositivos autónomos. Los alumnos tienen que redactar unos informes con los montajes realizados, problemas encontrados, soluciones adoptadas, etc. Son el reflejo de las experiencias en el laboratorio. Como trabajan en grupo, se les indica cómo deben comunicarse, tomar decisiones consensuadas, coordinar el trabajo, etc.

- Actividades de montaje. Los estudiantes construyen, bajo la supervisión de los profesores de la asignatura, un dispositivo a partir de las piezas que lo componen. En el curso 2014/15 se realizó el montaje de dos impresoras 3D (parte izquierda de la Figura 1) y en el curso 2015/16 se realizó el montaje de dos escáneres 3D (parte derecha de la Figura 1). Aunque existen manuales para su implementación, los estudiantes tienen que resolver, con la ayuda del profesor, situaciones inesperadas: erratas en los manuales, rotura de componentes, calibración, etc. Para ello, han de buscar soluciones, en ocasiones orientadas por el profesor, para solventar los problemas encontrados. Tienen que sopesar las diferentes opciones propuestas, argumentando cuál es la más idónea mediante el análisis de ventajas e inconvenientes. Además, requieren saber expresar su punto de vista adecuadamente para obtener la aprobación del grupo. En ocasiones también tienen que resolver conflictos derivados del trabajo en grupo, como la coordinación de tareas, la aceptación de responsabilidades, las decisiones erróneas, los errores individuales, etc. Se les orienta sobre cómo proceder durante las distintas situaciones generadas, tanto en la parte de conocimiento de los temas de la asignatura como a cómo comportarse en el grupo (MontoyaEstrada, 2012). 

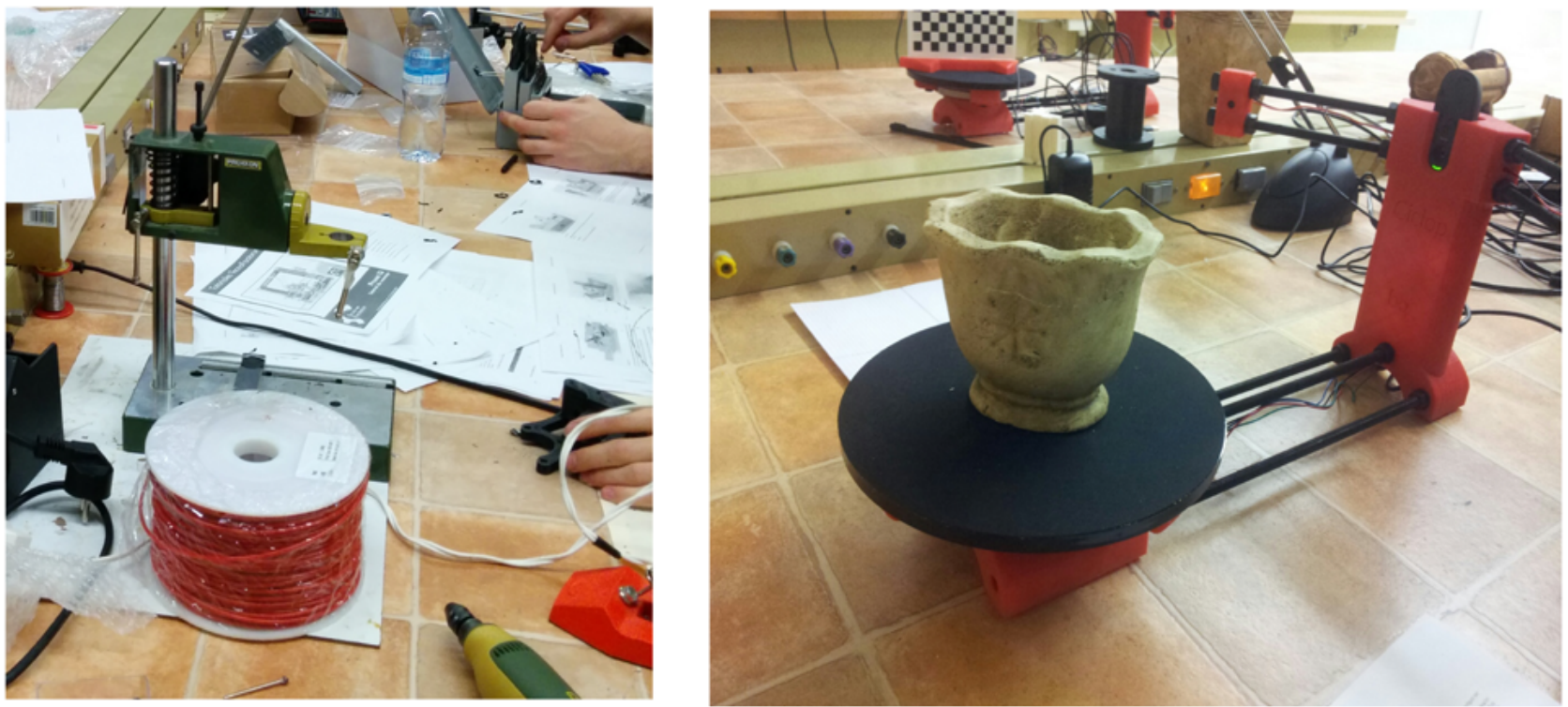

Figura 1. A la izquierda momento de montaje de la impresora 3D, a la derecha prueba de funcionamiento del escáner

- Actividades de desarrollo de un proyecto. Los estudiantes diseñan, planifican y desarrollan en su totalidad un sistema empotrado utilizando las herramientas vistas en clase. El sistema ha de ser completo y funcional y tiene que realizar alguna tarea por sí mismo. La elección del tema la realizan libremente los alumnos, algunos autónomamente y otros tras la orientación de los profesores de la asignatura. En clase se les proponen unos temas y les sirve o bien para escoger alguno, o bien para darles nuevas ideas que elaboran ellos posteriormente. El sistema desarrollado tiene todas las partes necesarias para su funcionamiento de forma autónoma: alimentación propia, interfaznecesariaparasuinstalación,configuración,operación,etc. Tambiénse valora el aspecto. La idea es que consigan un prototipo lo más parecido a lo que sería el producto final. Algunos ejemplos de proyectos presentados pueden observarse en la Figura. 2. entre ellos se tienen: un dispositivo para reconocer rostros montado en una Raspberry como control de acceso; un coche teledirigido usando Arduino, conexiones Bluetooth e imprimiendo las ruedas con la impresora 3D; un guante que mediante el movimiento de los dedos y la mano permite interaccionar con una Raspberry utilizando distintos sensores (flexores y sensores de presión/fuerza) y Arduino; un sistema hardware y software para jugar al LaserTag utilizando Arduino e infrarrojos para simular los disparos; un dispositivo para monitorizar el consumo de energía eléctrica en una vivienda; o un sistema domótico inalámbrico con control vía web.
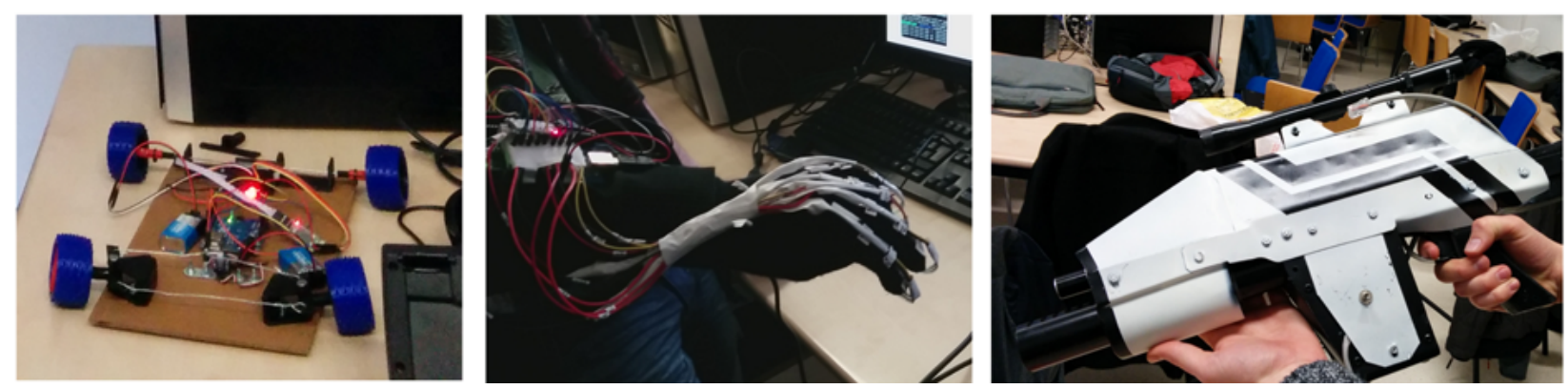

Figura 2. A la izquierda el coche teledirigido, en el medio el guante, a la derecha el sistema LaserTag 


\subsection{Seguimiento y evaluación de las actividades}

Para motivar a los estudiantes y que sean conscientes de la importancia que el profesorado otorga a esta metodología, todas las actividades realizadas en la asignatura constituyen la evaluación continua de la misma. Así, todas las tareas son entregables y no solo supervisadas durante su realización, sino evaluadas como resultados finales. Además, el sistema de evaluación forma parte del enunciado de las tareas a realizar con lo que los alumnos conocen no solo qué tienen que hacer, sino qué se va a valorar, cómo se va a realizar esa evaluación, cómo se deben planificar para ir alcanzando cada hito, cómo se pondera cada actividad e incluso información sobre el rango de puntuaciones según el grado de desempeño.

\section{Evaluación del experimento}

El sistema de evaluación utilizado va a medir el grado de adquisición y desempeño de las competencias indicadas. Por tanto, no se evalúa solo el resultado final, sino todo el proceso. Para ello es necesario hacer un seguimiento durante todo el desarrollo de la asignatura, lo que permite no solo valorar toda la experiencia, sino realizar una retroalimentación del desempeño de los estudiantes para que tengan oportunidad de mejora.

Una de las técnicas más habituales para obtener evidencias que permitan evaluar las competencias adquiridas está basado en la observación directa. Los cuestionarios o entrevistas también se emplean para determinar el grado de desempeño del alumno (Viles Diez, et al., 2013).

En la experiencia aquí descrita hemos usado ambas:

- Observación directa: comunicación entre los estudiantes y los profesores durante el desarrollo de las sesiones presenciales, así como la interacción por medios electrónicos.

- Cuestionarios: para medir el nivel de desempeño en los procesos mediadores durante la realización de las actividades. Mide tanto los procesos operativos como los estados emergentes anteriormente citados.

\subsection{Observación directa}

Durante el desarrollo de las actividades anteriormente indicadas, se han definido los siguientes aspectos a valorar por parte del profesorado durante su interacción con los alumnos, relacionados con los resultados de aprendizaje: 1) ¿Sabe trabajar en equipo? (observando su participación en la toma de decisiones, si coopera con los compañeros, etc.); 2) ¿Sabe analizar y resolver problemas (se tiene 
en cuenta su entendimiento del problema y cómo lo aborda); 3) ¿Sabe interpretar resultados y datos relevantes? (si sabe interpretar y gestionar los datos iniciales e intermedios); 4) ¿Sabe comunicarse vía oral y escrita? (en función de cómo actúa y los informes elaborados); 5) ¿Sabe aplicar los conocimientos de forma profesional? (si tiene en cuenta el desempeño profesional); 5) ¿Sabe elaborar y defender argumentos? (atendiendo especialmente a las discusiones en el grupo y a la presentación de resultados); y 6) ¿Sabe emitir juicios sobre temas de diversa índole? (para lo que se observa su comportamiento en las discusiones del equipo y en la presentación del trabajo final).

Estas cuestiones son tenidas en cuenta por los profesores y con una retroalimentación directa en el día a día, son incorporadas por los estudiantes en su desempeño diario. Debe entenderse que en este caso, al tratarse de 15 alumnos y 3 profesores, es sencillo establecer un diálogo y llevar a cabo una observación directa para aportar validez a esta técnica.

\subsection{Cuestionarios}

Los cuestionarios realizados han sido de dos tipos: encuestas y evaluación. Mediante ellos se trata de evaluar el desempeño tanto de los estudiantes como en los grupos.

\subsubsection{Encuesta a los alumnos}

Se ha utilizado una encuesta para evaluar las actividades desarrolladas y las operaciones que conllevan. Los ítems empleados se observan en la Tabla 1.

\begin{tabular}{|c|}
\hline 11. ¿Has realizado actividades similares en asignaturas anteriores? \\
\hline 12. Cuando se propusieron las actividades, ¿pensaste que serías capaz de realizarlas? \\
\hline I3. ¿Seguiste alguna metodología para realizar el proyecto? \\
\hline $\begin{array}{c}\text { 14. ¿Cuánto consideras que ayudaste, con tus aportaciones e intervenciones, a la consecución del objetivo } \\
\text { final? (100 si crees que lo hiciste todo tú y 0 si no hiciste nada) }\end{array}$ \\
\hline I5. De los integrantes de tu grupo, ¿quién aportó más ideas y solucionó más problemas? \\
\hline I6. ¿Tuvisteis que negociar en un momento dado qué decisión tomar? \\
\hline 17. Si negociasteis, describe cómo fue: ¿hubo posturas comprensivas, se dialogó, etc.? \\
\hline I8. En tu opinión, ¿quién se encargó de coordinar el reparto de tareas? \\
\hline 19. De las ideas propuestas para las distintas fases, ¿qué idea te pareció la más útil/original/reseñable? \\
\hline 170. Si hubieras decidido tú qué miembros componían tu grupo, ¿habrías elegido a todos los que lo \\
formaban?
\end{tabular}




\begin{tabular}{|c|}
\hline 113. En general, ¿trabajaste a gusto con los miembros de tu grupo? \\
\hline 114. ¿Surgió algún conflicto durante el montaje? \\
\hline I15. Si hubo un conflicto, ¿cómo se resolvió? \\
\hline I16. Respecto al otro grupo de montaje, ¿existió competitividad por ver quién lo hacía mejor/acababa \\
antes? \\
\hline I17. ¿Venías a clase motivado y "con ganas"? \\
\hline 118. ¿Dedicaste voluntariamente a la actividad más horas de las que estaban planificadas en la asignatura \\
por vuestro nivel de implicación en la tarea?
\end{tabular}

Tabla 1. Ítems utilizados en la encuesta a los alumnos.

\subsubsection{Autoevaluación}

Cada alumno ha tenido que realizar una encuesta de autoevaluación sobre el desempeño de las competencias (Tabla 2). El alumno debía puntuarse del 1 al 10 excepto en el primer ítem.

\begin{tabular}{|c|}
\hline I1. Aportación (0-100) \\
\hline I2. ¿Actuaste como líder? \\
\hline 13. ¿Ayudaste a la coordinación/organización? \\
\hline I4. ¿Propusiste ideas? \\
\hline I5. ¿Fuiste imprescindible? \\
\hline 16. ¿Crees que los miembros de tu grupo trabajaron a gusto contigo? \\
\hline 17. ¿Crees que tuvieron algún conflicto contigo? \\
\hline
\end{tabular}

Tabla 2. Ítems de la autoevaluación.

\subsubsection{Evaluación por parte del grupo}

Para todos los compañeros de su equipo de trabajo, cada estudiante tiene que responder a las mismas preguntas que tuvo que realizar para su autoevaluación.

\section{Resultados}

Durante las actividades de montaje, los integrantes de un grupo preguntaban y observaban cómo lo hacía el otro grupo. Hubo muy buena actitud por parte de ambos grupos, ya que cuando unos 
solucionaban un problema, avisaban al otro grupo. Cuando se encontraban con un problema, todos ayudaban, independientemente del grupo al que pertenecían. Hay que resaltar las soluciones creativas que proporcionaron ante distintas situaciones, como la rotura de piezas o los errores cometidos. Consiguieron resolver todos los problemas ideando soluciones.

La actitud de los alumnos, su compromiso con la asignatura, ha sido elevadísimo. No solo ha sido la impresión mediante la observación directa, sino también el resultado de las encuestas: el 100\% trabajó a gusto con los miembros de su equipo y venía a clase motivado; cerca del 70\% le dedicó voluntariamente más horas a la actividad; el 100\% recomendaría la asignatura a un compañero; las opiniones sobre la metodología fueron todas muy positivas.

Cabe destacar que el año 2014/15, cuando se realizó el montaje de dos impresoras 3D, una por cada grupo, la dinámica de los grupos fue distinta: un grupo tenía un claro líder (los alumnos lo identificaron en las encuestas), mientras que en el otro todos compartieron el liderazgo (según las encuestas). Durante la experiencia del curso 2015/16 los alumnos experimentaron un cambio muy significativo en su actitud y motivación por la asignatura y por los conocimientos adquiridos, motivados por la nueva experiencia de enfrentarse a un proyecto "real" por sí mismos y poder construirlo.

Se observa una evolución de los alumnos durante las prácticas, que pasan de seguir meramente los guiones y entregar tareas con soluciones no muy adecuadas a tener una mayor implicación y adquirir más responsabilidad, con lo que al final se obtienen proyectos completos que ofrecen soluciones de alta calidad. Además de también un cambio importante de actitud por parte de los alumnos especialmente motivados ante los métodos más prácticos, basados en la adquisición de conocimientos mediante la experiencia.

En cuanto a la evaluación, los estudiantes valoraron positivamente el trabajo de sus compañeros de grupo, considerando que ayudaron tanto en la coordinación como proponiendo ideas y realizando las distintas tareas. En la Figura 3 se observa la distribución de la aportación personal considerada por cada estudiante frente a la media que le asignan los miembros del equipo para el año 2014/15. Cabe destacar que uno de los grupos (estudiantes 1, 4, 9, 10, 13 y 14) se repartieron equitativamente su dedicación mientras que el resto asignaron la dedicación que les pareció oportuno. En este último grupo los miembros del equipo valoran al resto por encima de la valoración personal de cada uno y tiene especial relevancia el papel del líder comentado con anterioridad (E3). Esto refleja dos formas diferentes de trabajar en equipo, distribuyendo el trabajo o con personas que lo lideran especialmente. 


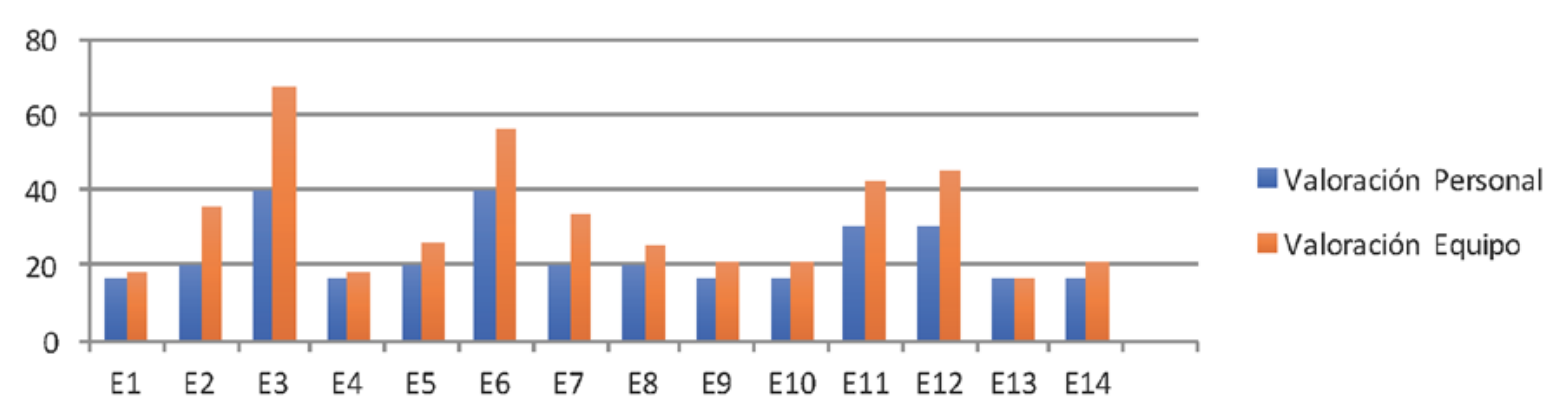

Figura 3. Comparativa de autoevaluaciones y evaluación del grupo

\section{Conclusiones}

La experiencia presentada muestra una metodología para conseguir que los estudiantes adquieran las competencias necesarias para aumentar su empleabilidad y su autoempleo, en función de un conjunto de competencias conocidas.

Basándose en modelos existentes, se propone un procedimiento para la adquisición de competencias donde se identifican los fundamentos sobre los que se adquieren las competencias y que permiten su evaluación. Este modelo se ha empleado satisfactoriamente en la asignatura de Arquitecturas Específicas y Empotradas de 4. ${ }^{\circ}$ de Grado en Ingeniería Informática por dos cursos consecutivos.

El trabajo presentado es una acción innovadora en el campo de la enseñanza, ya que se expone a los alumnos ante una situación real de futuro empleo donde deberán desarrollar un proyecto completo en todas sus fases e implementarlo físicamente, al menos a nivel de prototipo. Los alumnos tienen que afrontar todos los problemas de la planificación, búsqueda y elección de materiales, desarrollo, implementación, modificaciones, etc., característicos de los proyectos reales.

\section{Referencias}

Blank, W. (1997). Authentic instruction. In W. E. Blank \& S. Harwell (Eds.), Promising practices for connecting high school to the real world (pp. 15-21). Tampa, FL: University of South Florida. (ERIC Document Reproduction Service No. ED407586)

Blumenfeld, P. C., Soloway, E., Marx, R. W., Krajcik, J. S., Guzdial, M., \& Palincsar, A. (1991). Motivating Project-Based Learning: Sustaining the Doing, Supporting the Learning. Educational Psychologist, 26(34), 369-398. doi:http://dx.doi.org/10.1080/00461520.1991.9653139

Bottoms, G., \& Webb, L. D. (1998). Connecting the curriculum to "real life." Breaking Ranks: Making it happen. Reston, VA: National Association of Secondary School Principals. (ERIC Document Reproduction Service No. ED434413) 
Clark, B. R. (1998). Creating Entrepreneurial Universities: Organizational Pathways of Transformation: IAU Press.

Crespo, E., Alba-Elías, F., González-Marcos, A., Ordieres-Meré, J., \& Castejón-Limas, M. (2011). Project Management Learning in a Collaborative Distant Learning Context - An Actual On-going Experience. Paper presented at the 3rd International Conference on Computer Supported Education, Noordwijkerhout, The Netherlands.

Dickinson, K. P., Soukamneuth, S., Yu, H. C., Kimball, M., D'Amico, R., Perry, R., .. P., C. S. (1998). Providing educational services in the Summer Youth Employment and Training Program [Technical assistance guide]. Washington, DC: U.S.: Department of Labor, Office of Policy \& Research (ERIC Document Reproduction Service No. ED420756)

García-Peñalvo, F. J., Cruz-Benito, J., Conde, M. Á. \& Griffiths, D. (2014, 22-25 Oct. 2014). Virtual placements for informatics students in open source business across Europe. Paper presented at the 2014 IEEE Frontiers in Education Conference (FIE) Proceedings, Madrid, Spain. doi:https://doi.org/10.1109/ FIE.2014.7044411

García-Peñalvo, F. J. (2015). Entrepreneurial and problem solving skills in software engineers. Journal of Information Technology Research, 8(3), 4-6.

García-Peñalvo, F. J. (2016). La tercera misión. Education in the Knowledge Society, 17(1), 7-18. doi:http:// dx.doi.org/10.14201/eks2016171718

García-Peñalvo, F. J., Cruz-Benito, J., Griffiths, D., \& Achilleos, A. P. (2015). Tecnología al servicio de un proceso de gestión de prácticas virtuales en empresas: Propuesta y primeros resultados del Semester of Code. IEEE VAEP-RITA, 3(1), 52-59.

Gomes-Pires, J. A., García-Peñalvo, F. J., Marinho-Sampaio, J. H., \& Martínez-Vázquez, R. M. (2013). Framework Entrepreneurship Process. In S. Anna, K. Waldemar \& P. Patricia Ordóñez de (Eds.), Academic Entrepreneurship and Technological Innovation: A Business Management Perspective (pp. 228254). Hershey, PA, USA: IGI Global.

González, M. R. (2008). El enfoque por competencias en el EEES y sus implicaciones en la enseñanza y el aprendizaje. Tendencias Pedagógicas, 13(2008), 79-105.

Jun, H. (2010). Improving undergraduates' teamwork skills by adapting project-based learning methodology. Paper presented at the 5th International Conference on Computer Science and Education (ICCSE), Hefei, China. doi:https://doi.org/10.1109/iccse.2010.5593527 
Michavila, F., Martín-González, M., Martínez, J. M., García-Peñalvo, F. J., \& Cruz-Benito, J. (2016). Barómetro de Empleabilidad y Empleo de los Universitarios en España, 2015 (Primer informe de resultados). Madrid, Spain: Observatorio de Empleabilidad y Empleo Universitarios.

Montoya-Estrada, J. O. (2012). Metodología para medir y evaluar individualmente el trabajo en equipo. Ingeniería y Sociedad, 4, 1-13.

Musa, F., Mufti, N., Latiff, R. A., \& Amin, M. M. (2011). Project-based Learning: Promoting Meaningful Language Learning for Workplace Skills. Procedia - Social and Behavioral Sciences, 18, 187-195. doi: http://dx.doi.org/10.1016/j.sbspro.2011.05.027

Rae, D. (2007). Connecting enterprise and graduate employability: Challenges to the higher education culture and curriculum? Education + Training, 49(8/9), 605-619. doi:http://dx.doi. org/10.1108/00400910710834049

Thomas, J. W. (2000). A review of research on project-based learning. San Rafael, California: Autodesk Foundation.

Universidad-de-León. (2014). Informe de los alumnos de nuevo ingreso en el Grado en Ingeniería Informática para el curso 2014/15. León: Universidad de León.

Vilalta, J. M. (2013). La tercera misión universitaria. Innovación y transferencia de conocimientos en las universidades españolas. Madrid: Studia XXI. Fundación Europea Sociedad y Educación.

Viles Diez, E., Zárraga-Rodríguez, M., \& Jaca García, C. (2013). Herramienta para evaluar el funcionamiento de los equipos de trabajo en entornos docentes. Intangible Capital, 9(1), 281-304. 